\title{
Method of Suppressing the Side Lobe of a Tapered Short Leaky Wave Antenna
}

\author{
Jin-Wei Wu, Chien-Jen Wang, Senior Member, IEEE, and Christina F. Jou
}

\begin{abstract}
This letter demonstrates a method for suppressing the side lobe of a tapered short leaky wave antenna (LWA). The proposed LWA contains a tapered microstrip radiator with a shorting pin and two rectangular slots. This design of the two slots and the shorting pin can interfere with the current distribution of tapered LWAs to suppress the radiation of the side lobe. A matching stub is added along the feeding line to achieve impedance matching. According to the measured results, the proposed design improves the side lobe level (SLL) from $0 \mathrm{~dB}$ for the tapered short LWA (about $1.5 \lambda_{0}$ at $4.5 \mathrm{GHz}$ ) to $-6.13 \mathrm{~dB}$ for the proposed LWA at $6.4 \mathrm{GHz}$. The 7-dB impedance bandwidth is about $1.6 \mathrm{GHz}$, and the scanning range is about $43^{\circ}$.
\end{abstract}

Index Terms-Beam scanning, leaky wave antenna (LWA), side lobe, tapered antenna.

\section{INTRODUCTION}

$\mathbf{I}$ N 1979, Menzel presented the leaky wave antenna (LWA), which used an asymmetric feed line to excite the first higher order mode [1]. Because the LWA is operated in the first higher order mode [2], it possesses the advantages of having a frequency scanning capability, narrow beamwidth, and high gain. However, the width of the LWA, the thickness of the substrate, and the dielectric constant control the radiation bandwidth. To improve the radiation bandwidth, some researchers proposed the tapered structure LWA, as in [3] and [4]. A tapered LWA works by using sections with different widths and lengths to excite different operating frequency bands. The tapered LWA then combines these to increase the impedance bandwidth. Although broad impedance bandwidth can thus be achieved, the problem with tapered LWAs is that there is a spurious side lobe generated by the backward radiation.

This side lobe often occurs in the LWA, and especially the short LWA. Therefore, several researchers have tried to solve this problem [5]-[8]. In [5], the LWA array method reduced the reflected wave power and increased the power gain. In [6], Shih added an active feedback synthesizer and a closed loop to greatly decrease the side lobe level (SLL). A LWA with an aperture-fed antenna offered another radiated path for the reflected wave from the open end of the LWA to suppress the side lobe [7]. In 2008, Li proposed a parasitic topology utilizing two microstrip patches with short-circuit edges [8]. This topology coupled the radiation power of the LWA to the ground plane to

Manuscript received August 12, 2009; revised September 23, 2009. First published October 13, 2009; current version published November 06, 2009. This work was supported in part by the National Science Council of Taiwan under Grants NCS 96-2221-E024-001 and 97-2221-E-009-002.

J.-W. Wu and C. F. Jou are with the Department of Communication Engineering, National Chiao Tung University, Hsinchu 30010, Taiwan.

C.-J. Wang is with the Department of Electrical Engineering, National University of Tainan, Tainan 700, Taiwan (e-mail: cjwang@ mail.nutn.edu.tw).

Digital Object Identifier 10.1109/LAWP.2009.2034474

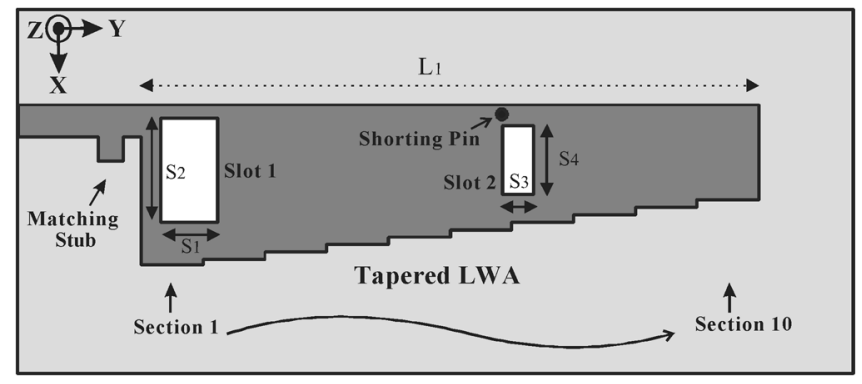

Fig. 1. Structure of the proposed short LWA.

suppress the back lobe. All these topologies achieved good results in suppressing the side lobe.

This letter presents a novel method to solve the spurious side lobe problem of a tapered short LWA (approximately only $1.5 \lambda_{0}$ at $4.5 \mathrm{GHz}$ ). The proposed LWA is composed of two rectangular slots and a shorting pin on the tapered short LWA. The measured results show that this design suppresses the side lobe over $5 \mathrm{~dB}$ at higher frequency bands $(6.0 \sim 6.4 \mathrm{GHz})$, achieves a main lobe scanning angle of about $43^{\circ}$ (from $14^{\circ}$ to $57^{\circ}$ between 4.6 to $6.4 \mathrm{GHz}$ ), and yields a $7-\mathrm{dB}$ impedance bandwidth of about $1.6 \mathrm{GHz}$ from 4.58 to $6.18 \mathrm{GHz}$. Sections II and III give details of the antenna design and results.

\section{ANTENNA DESIGN}

Fig. 1 shows the structure of the proposed short-length LWA. The antenna is printed on a 1.6-mm-thick FR4 substrate. The dielectric constant and the loss tangent are 4.1 and 0.02 , respectively. It is fed by a microstrip feed line with a matching stub. A tapered microstrip radiator with a shorting pin and two rectangular slots (Slots 1 and 2) make up the antenna. The total length $L \mathrm{~B}_{1}$ of the antenna is $10.0 \mathrm{~cm}$ (about $1.5 \lambda \mathrm{B}_{0}$ at $4.5 \mathrm{GHz}, \lambda \mathrm{B}_{0}$ is the free-space wavelength). The length of each section of the tapered LWA is $10.0 \mathrm{~mm}$. Table I lists the width of each section. The sizes of $S \mathrm{~B}_{1 \mathrm{~B}} \times S \mathrm{~B}_{2 \mathrm{~B}}\left(\right.$ Slot 1) and $S \mathrm{~B}_{3 \mathrm{~B}} \times S \mathrm{~B}_{4 \mathrm{~B}}$ (Slot 2) are $6.0 \times 12.0 \mathrm{~mm}^{2}$ and $6.0 \times 7.5 \mathrm{~mm}^{2}$, respectively. This section utilizes an Ansoft High Frequency Structure Simulator to simulate the proposed antenna.

Hong uses the tapered leaky wave antenna to increase impedance bandwidth [4]. However, the tapered short LWA excites serious side lobes, even replacing the main lobe with a side one at higher frequencies. Fig. 2 displays the measured normalized radiation patterns of the conventional tapered short LWA. As can be seen from Fig. 2, the gain of the side lobe greatly increases as the frequency increases, and the side lobe is equal to the main one at $6.4 \mathrm{GHz}$.

In order to suppress the side lobe, this study proposes the method of using two slots (Slots 1 and 2) and a shorting pin. 


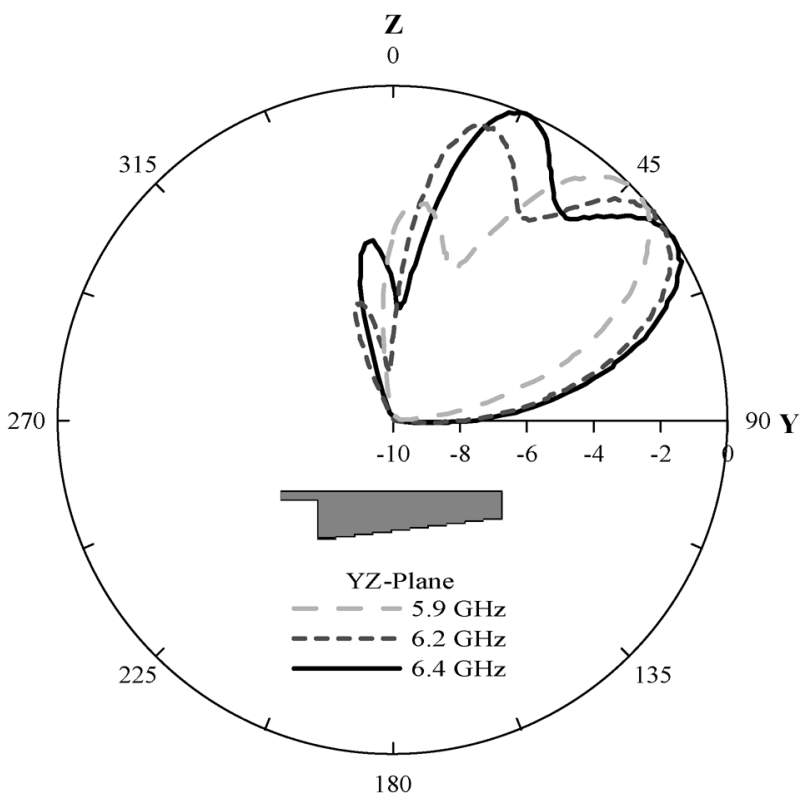

Fig. 2. Measured normalized radiation patterns of the conventional tapered short LWA.

TABLE I

DiMENSIONS OF THE PROPOSED TAPERED SHORT LWA

\begin{tabular}{|l|c|c|c|}
\hline Width of Section 1 & $15.0 \mathrm{~mm}$ & Width of Section 6 & $11.7 \mathrm{~mm}$ \\
\hline Width of Section 2 & $14.5 \mathrm{~mm}$ & Width of Section 7 & $11.0 \mathrm{~mm}$ \\
\hline Width of Section 3 & $13.8 \mathrm{~mm}$ & Width of Section 8 & $10.3 \mathrm{~mm}$ \\
\hline Width of Section 4 & $13.1 \mathrm{~mm}$ & Width of Section 9 & $9.6 \mathrm{~mm}$ \\
\hline Width of Section 5 & $12.4 \mathrm{~mm}$ & Width of Section 10 & $8.9 \mathrm{~mm}$ \\
\hline
\end{tabular}

Slot 1 suppresses the excitation of the dominant mode. Slot 2 affects the reflected wave and perturbs the current distribution at the end of the tapered short LWA, suppressing the radiation of the side lobe. To reduce the side lobe further, the shorting pin near Slot 2 guides the power at the end of LWA to the ground. Furthermore, a matching stub is added along the feeding line to achieve impedance matching.

Fig. 3 shows the simulated radiation patterns in the YZ-plane at $6.0 \mathrm{GHz}$. These methods successfully suppress the SLL, as shown in Fig. 3. Fig. 4 plots the simulated surface current distributions of the tapered short LWA at $6.0 \mathrm{GHz}$ with Slot 1 and with Slots 1 and 2. Fig. 4(a) shows that the strong power of the current distribution at the end of the tapered short LWA generates a significant side lobe. When Slot 1 is embedded on the LWA, although the current distribution in Fig. 4(b) can be changed, the power at the end of the LWA still radiates a large side lobe. However, Fig. 4(c) shows that with Slots 1 and 2 embedded, the path and the direction of the current at the end of the LWA are varied, and the side lobe of the LWA with Slot 1 alone is separated into two weak side lobes, as shown in Fig. 3. The shorting pin, located near Slot 2, reduces the power at the end of the LWA with Slots 1 and 2. Because the shorting pin guides the power at the end of LWA to the ground, the pin reduces the side lobe, as shown in Fig. 4(d). This method successfully suppresses the side lobe of the conventional tapered short LWA.

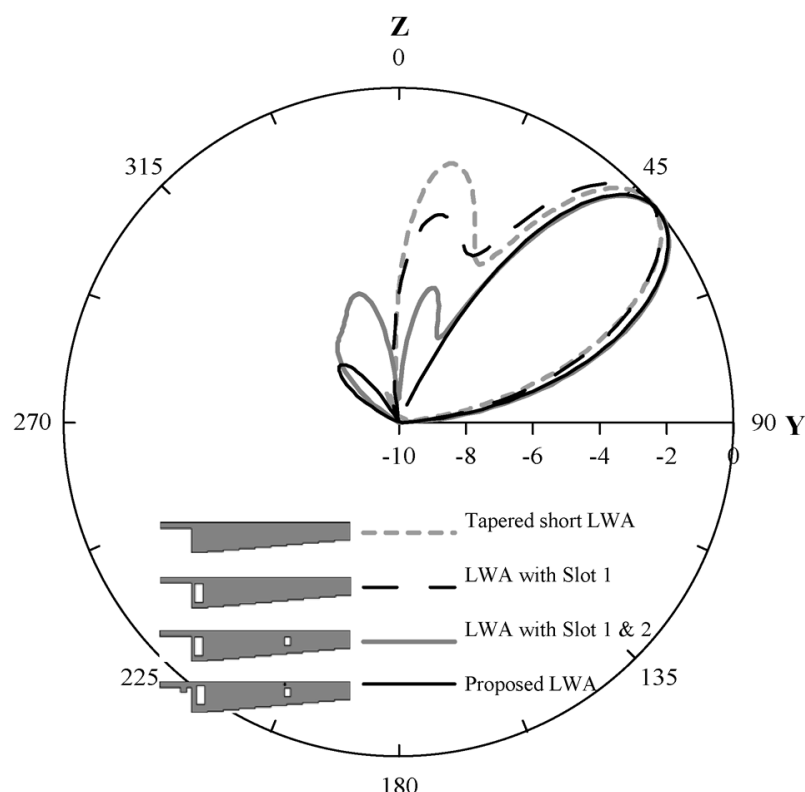

Fig. 3. Simulated radiation patterns in the YZ-plane at $6.0 \mathrm{GHz}$.

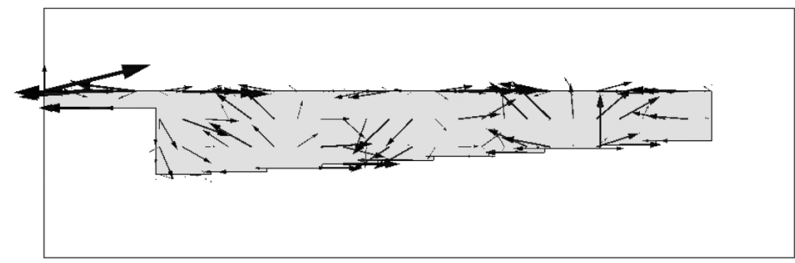

(a)

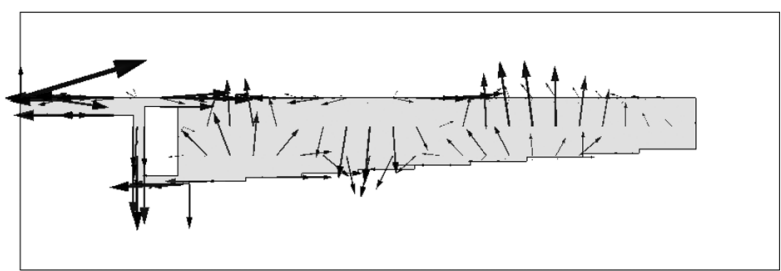

(b)

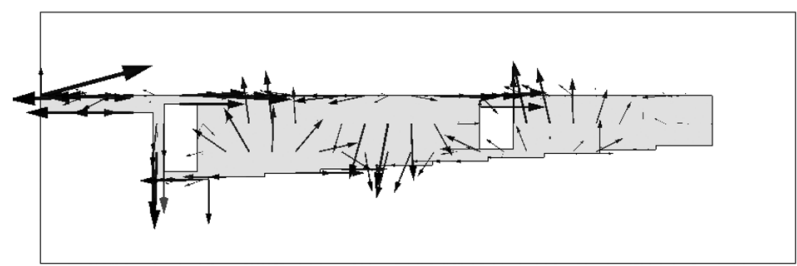

(c)

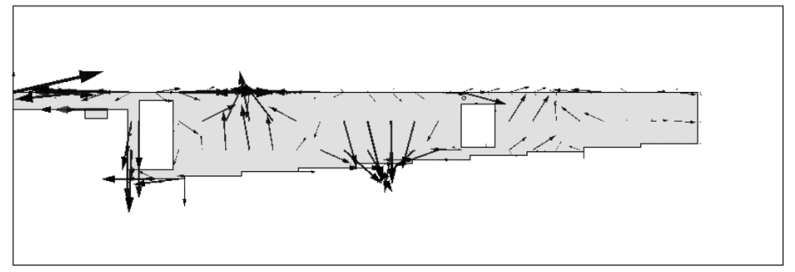

(d)

Fig. 4. Simulated surface current distributions at $6.0 \mathrm{GHz}$ : (a) tapered short LWA; (b) LWA with Slot 1; (c) LWA with Slots 1 and 2; (d) proposed LWA.

Figs. 5 and 6 show the effects of the distance between Slots 1 and 2 on the radiation pattern in the YZ-plane and on the 


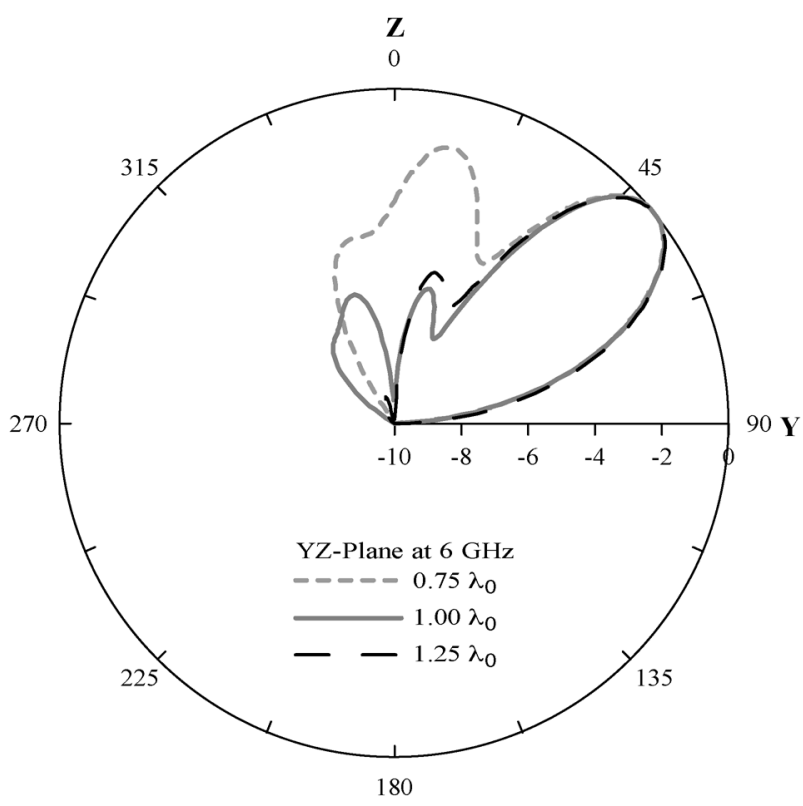

Fig. 5. Simulated radiation patterns of different positions of Slot 2 in YZ-plane at $6.0 \mathrm{GHz}$.

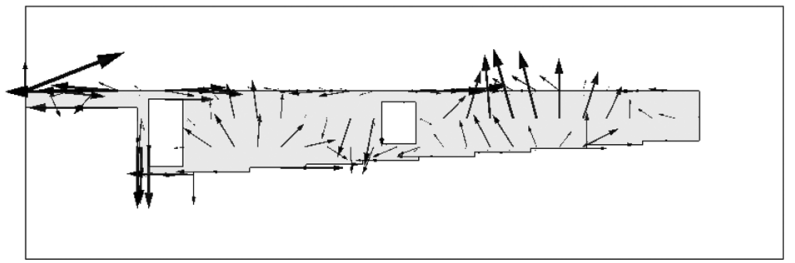

(a)

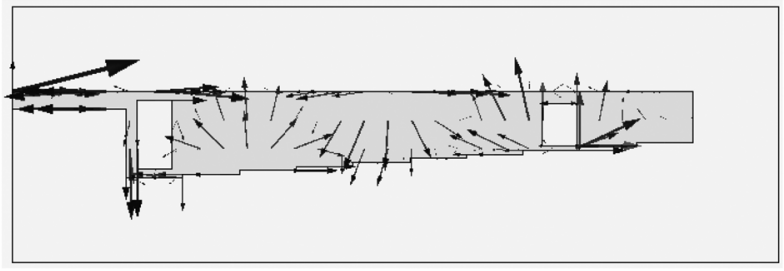

(b)

Fig. 6. Simulated surface current distribution when varying the distance between Slots 1 and Slot 2 at $6.0 \mathrm{GHz}$ : (a) $0.75 \lambda_{0}$; (b) $1.25 \lambda_{0}$.

current distribution at $6.0 \mathrm{GHz}$, as the position of Slot 1 was fixed. At a distance of $0.75 \lambda_{0}$, where $\lambda_{0}$ is the wavelength at $6.0 \mathrm{GHz}$, the power at the end of the LWA is larger in Fig. 6(a), thus increasing the SLL. However, when the distance is longer than $1.0 \lambda_{0}$, the power and the direction of the current at the end of the LWA varies, as shown in Figs. 4(c) and 6(b), reducing the SLL, as shown in Fig. 5. Based on these simulated results, when the distance is shorter than $1 \lambda_{0}$, the intensity of the current distribution decreases between Slots 1 and 2 and increases at the antenna end, enhancing the SLL. At a distance longer than $1 \lambda_{0}$, the power and the direction of current at the end of the LWA suppress the SLL. Therefore, the seat of Slot 2 can interfere with the power and direction of the current at the end to vary the SLL.

Fig. 7 shows the simulated radiation patterns for various placements of the shorting pin. When the shorting pin is set in Seat-A, the SLL is improved. This is because the power of the

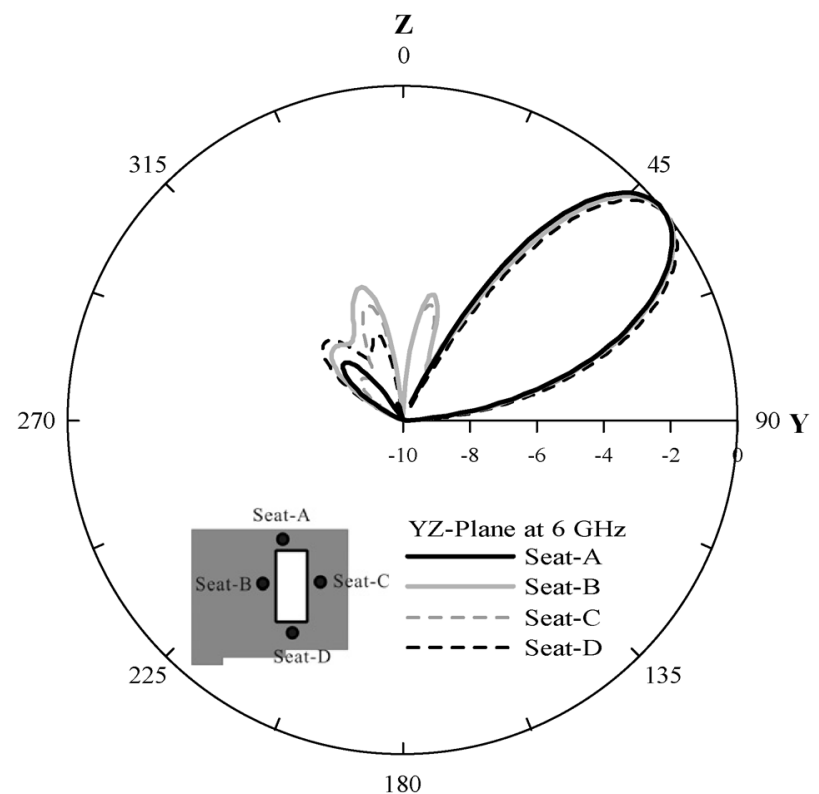

Fig. 7. Simulated radiation patterns of different seats of shorting pin in YZ-plane at $6.0 \mathrm{GHz}$.

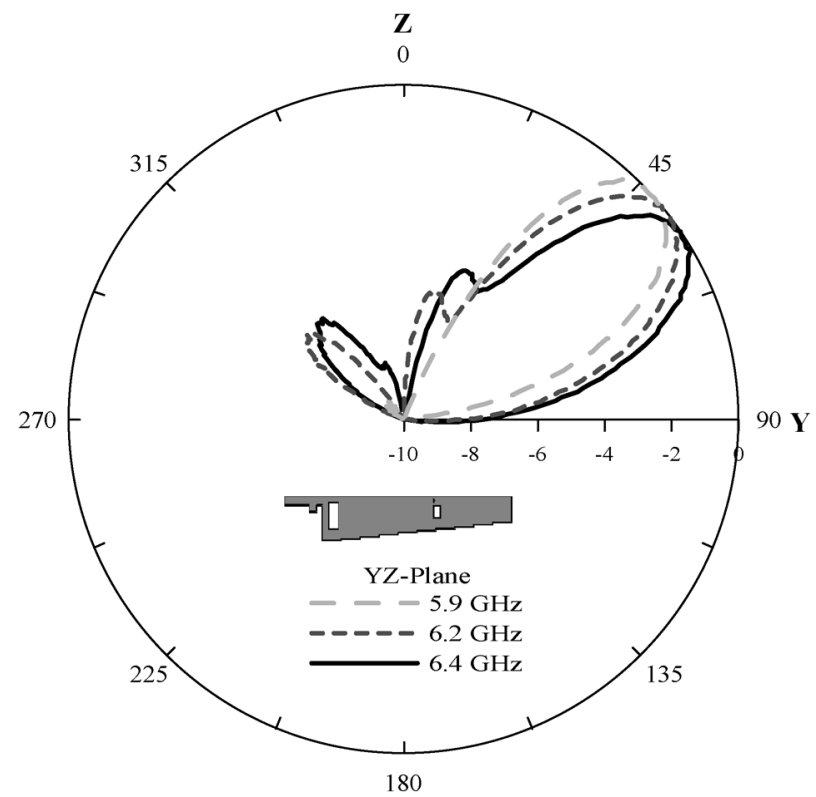

Fig. 8. Measured normalized radiation patterns of the proposed LWA.

current distribution near Seat-A, shown in Fig. 4(c), is greater than other placements, and the power of the side lobe is guided to the ground, suppressing the SLL when the shorting pin is set in this position.

\section{RESULTS}

Fig. 8 shows the measured normalized radiation patterns of the proposed LWA at 5.9, 6.2, and $6.4 \mathrm{GHz}$. Comparing the measured radiation pattern of Fig. 2 with that of Fig. 8 reveals that the tapered short LWA and the proposed LWA have similar radiation angles and 3-dB radiation beamwidths at $6.2 \mathrm{GHz}$. However, the SLL of the proposed LWA significantly improves, from 0 to $-6.13 \mathrm{~dB}$ at $6.4 \mathrm{GHz}$. The main lobe scanning angle of the proposed LWA is from $14^{\circ}$ to $57^{\circ}$ between 4.6 


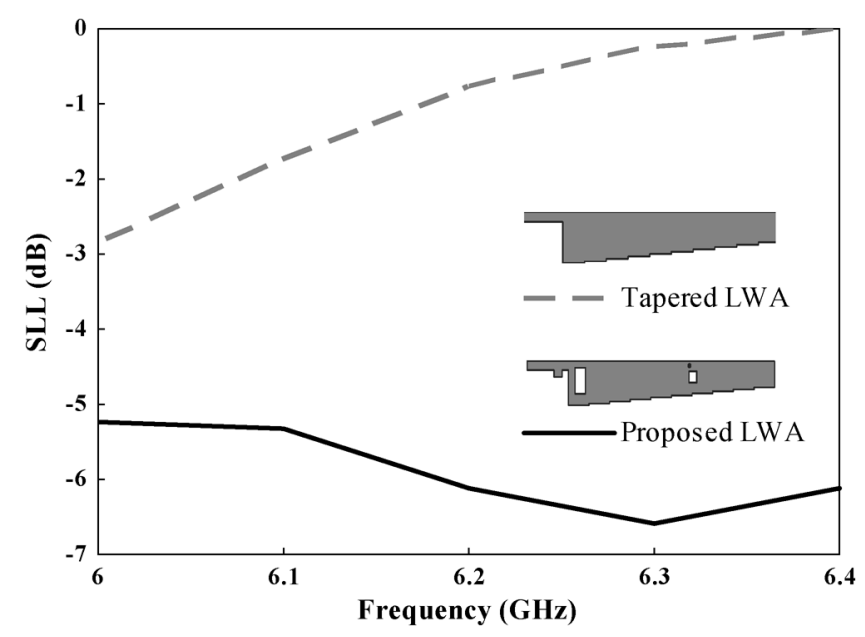

Fig. 9. Comparison of the measured SLL of tapered LWA and proposed LWA.

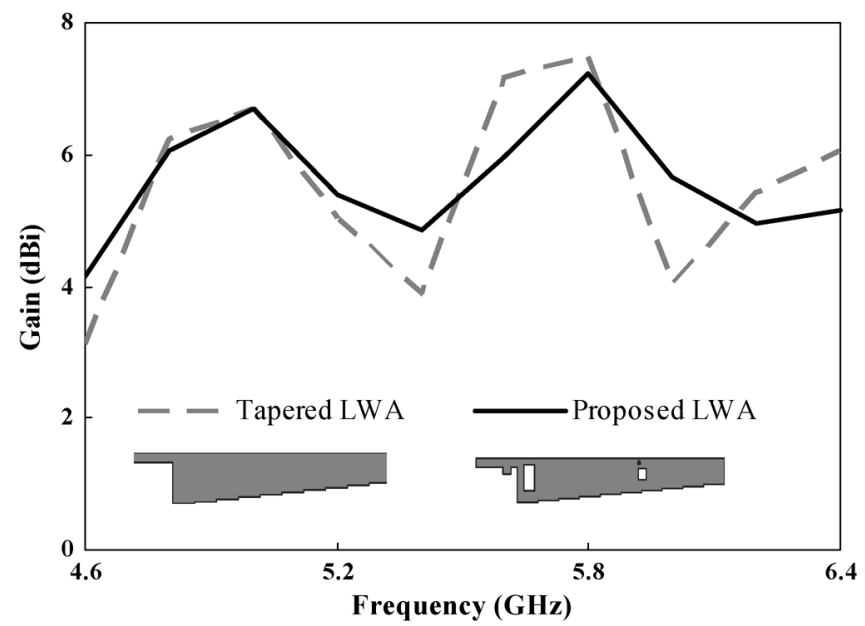

Fig. 10. Comparison of measured maximum gains of the tapered LWA and the proposed LWA.

to $6.4 \mathrm{GHz}$. Fig. 9 exhibits the measured SLL of the proposed LWA, which is less than $-5 \mathrm{~dB}$, and the variation of the SLL is independent of the frequency. Fig. 10 illustrates the measured maximum measured gains of the tapered and the proposed LWA. The proposed method slightly affects the variation in maximum gain of the LWA, and the gains are larger than $4 \mathrm{dBi}$ from 4.6 to $6.4 \mathrm{GHz}$. Using these methods not only improves the SLL, but also keeps the radiated power of the main lobe. Fig. 11 plots the simulated and measured return losses. The 7-dB impedance bandwidth of the measured results is about $1.6 \mathrm{GHz}$ from 4.58 to $6.18 \mathrm{GHz}$. Although the bandwidth of the proposed LWA is narrower than that of the conventional tapered LWA (see Fig. 3), the proposed LWA can largely suppress side lobes, preventing them from replacing the main lobe at higher frequencies.

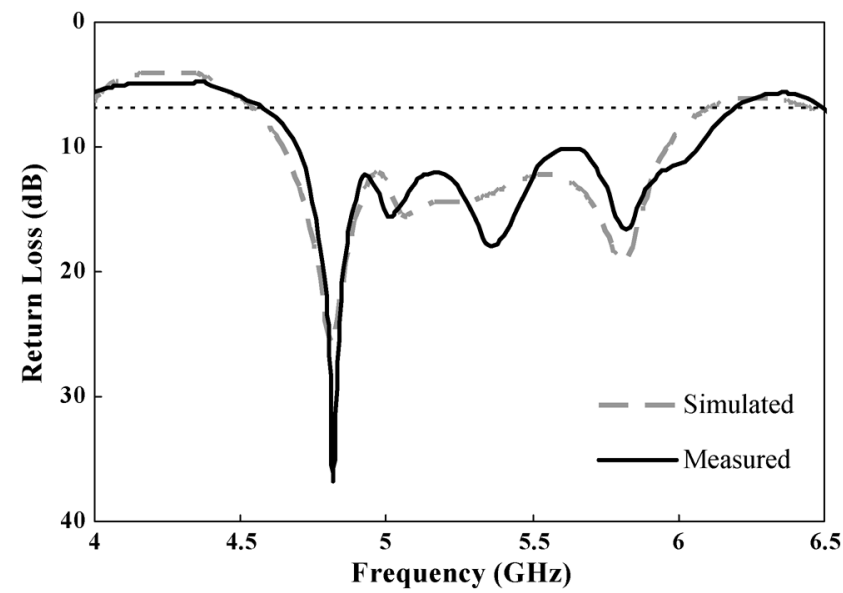

Fig. 11. Simulated and measured return losses of the proposed LWA.

\section{CONCLUSION}

This letter proposed a method to suppress the significant side lobes excited by the conventional tapered short LWA. By embedding two slots and a shorting pin, the method can change the current distribution at the end of the tapered short LWA at higher frequencies to reduce the radiation of a side lobe. Based on the measured results, the SLL of the proposed LWA remains less than $-5 \mathrm{~dB}$ from 6.0 to $6.4 \mathrm{GHz}$. The scanning range covers $43^{\circ}$ from $14^{\circ}$ to $57^{\circ}$, and the impedance bandwidth is about $30 \%$ at the center frequency of $5.38 \mathrm{GHz}$. Compared to the conventional tapered short LWA, the proposed LWA not only suppresses the side lobe, but also retains a wide impedance bandwidth. Furthermore, this method does not use any parasitic element or circuit, avoiding a larger antenna size.

\section{ACKNOWLEDGMENT}

The authors are grateful to the National Center for Highperformance Computing and the Chip Implementation Center (CIC) of National Applied Research Laboratories, Taiwan, for support with regard to simulation software and facilities.

\section{REFERENCES}

[1] W. Menzel, "A new travelling-wave antenna in microstrip," Archiv. Electrnik, Ubertrag Tech., pp. 137-140, Apr. 1979, Band 33.

[2] A. A. Oliner, "Leakage from higher modes on microstrip line with application to antennas," Radio Sci., vol. 22, no. 6, pp. 907-910, Nov. 1987.

[3] V. Nalbandian and C. S. Lee, "Tapered leaky-wave ultra wide-band microstrip antenna," in Proc. IEEE AP-S Int. Symp., 1999, pp. 1236-1239.

[4] W. Hong, T. L. Chen, C. Y. Chang, J. W. Sheen, and Y. D. Lin, "Broadband tapered microstrip leaky-wave antenna," IEEE Trans. Antennas Propag, vol. 51, no. 8, pp. 1922-1928, Aug. 2003.

[5] C. J. Wang, C. F. Jou, J. J. Wu, and S. T. Peng, "Radiation characteristic of active frequency-scanning leaky-mode antenna arrays," IEIEC Trans. Electron., vol. E82-C, no. 7, pp. 1223-1228, Jul. 1999.

[6] Y. C. Shih, S. K. Chen, C. C. Hu, and C. F. Jou, "Active feedback microstrip leaky wave antenna-synthesizer design with suppressed back lobe radiation," Electron. Lett., vol. 35, no. 7, pp. 513-514, Apr. 1999.

[7] C. J. Wang, H. L. Guan, and C. F. Jou, "A novel method for short leakywave antennas to suppress the reflected wave," Microw. Opt. Technol. Lett., vol. 36, no. 2, pp. 129-131, Jan. 2003.

[8] Y. X. Li, Q. Xue, E. K. N. Yung, and Y. L. Long, "Radiation patterns of microstrip leaky-wave antenna with parasitic elements," Microw. Opt. Technol. Lett., vol. 50, no. 6, pp. 1565-1567, Jun. 2008. 